This is a pre-copyedited, author-produced PDF of an article accepted for publication in Teaching Mathematics and its Applications following peer review. The version of record Maria Jacob, Eabhnat Ni Fhloinn, A quantitative, longitudinal analysis of the impact of mathematics support in an Irish university, Teaching Mathematics and its Applications: An International Journal of the IMA, , hry012, https://doi.org/10.1093/teamat/hry012 is available online at: https://academic.oup.com/teamat/advancearticle/doi/10.1093/teamat/hry012/4995148?guestAccessKey=850e91a0-f599-4885-941f$9 a d 53 f 165 a b 2$

\title{
A quantitative, longitudinal analysis of the impact of mathematics support in an Irish university
}

\author{
Maria Jacob and Eabhnat Ní Fhloinn
}

\section{Dublin City University}

\begin{abstract}
Mathematics support has become embedded in a large number of higher education institutes in Ireland and UK in particular. Measuring the impact of such support is a challenging task, which can be attempted either qualitatively, through the use of surveys or focus groups, or quantitatively, looking at data such as number of visits and subsequent exam performance. Here, we consider a quantitative analysis of the impact of a mathematics support centre in an Irish university, based on data gathered over the past 12 years. A binary logistic regression was carried out which showed that, when prior mathematical achievement and module studied were kept constant, the odds of a student who attended mathematics support once passing their module were 1.63 times higher than for one who had never engaged with the service. The odds for those who attended 15 or more times were almost 14 times higher. This study also showed that there was a significant difference between those who never engaged with mathematics support and those who attended once, meaning that such cohorts should be considered differently, which has not traditionally been done in other studies conducted to this point.
\end{abstract}

\section{Introduction}

Measuring the impact of mathematics support is a difficult process, given that it should 
by its nature support the traditional lecture-tutorial format for students who need additional help. Isolating the impact of this support from students' learning experiences in lectures, tutorials, lab classes, online homework and so on is challenging. However, as mathematics support becomes embedded in higher education over a longer period of time, longitudinal data and larger sample sizes mean that a more reliable picture of this impact can be investigated from a quantitative point of view.

The Mathematics Learning Centre (MLC) in Dublin City University (DCU) was set up in February 2004 with the aim of providing additional assistance to all undergraduate students undertaking any mathematics module as part of their degree. In 2006, some early indications of the effectiveness of the MLC were reported by Dowling \& Nolan (2006). This work presented evidence that the MLC had a positive impact on student retention and that the pass rates of First Science students (who can go on to specialize in programmes such as Biotechnology, Environmental Science and Health, Analytical Science or Chemical and Pharmaceutical Sciences) and at-risk students were higher for those who had attended the MLC than those who had not.

While the findings at the time were not conclusive, they were important in validating the role of and acquiring funding for the MLC, which is in operation to date. In fact, in 2014/2015, the MLC was relocated to the ground floor of the Library, to a location which is more accessible, more comfortable and has a higher seating capacity. Given this recent upgrade and the duration for which the MLC has been in operation, it is an appropriate time to conduct a longitudinal study of the impact of mathematics support within DCU to date. Therefore, in this paper, we will address the following research questions: 
(1) When controlling for factors such as prior mathematical background and module studied, what impact does usage of mathematics support services have upon student success in final examinations?

(2) Given the varying levels of support that may be optimal for different students, is it appropriate to consider even a single visit as engaging with mathematics support?

\section{Literature review}

A number of studies on the effectiveness of mathematics support have been conducted in Ireland and UK, with some relying on qualitative data such as surveys, focus groups and interviews and others taking a more quantitative approach, studying attendance rates and examination performance. A comprehensive review of all of these studies is outside the scope of this paper, but can instead be found in the study by Matthews et al. (2013). Here, we will focus in detail on some of the quantitative studies conducted to date.

Patel \& Little (2006) looked at pass rates of students who had availed of the MLC service versus those who had not and found that $92 \%$ of mathematics-related passes were associated with MLC use in comparison to $88 \%$ passes related to non-use of the MLC.

Pell \& Croft (2008) analysed data from five first-year modules in the Engineering department in Loughborough University. They found that $20 \%$ of students in all but one module attended the MLC between 2 and 9 times, whereas 3-8\% of students used it 10 times or more. Surprisingly, however, $35 \%$ of students who had achieved an $\mathrm{A}^{*}$ attended the support more than once and from this the authors concluded that the facility was being used more by students seeking excellence than by less-able students looking to avoid failure. Moreover, it is noteworthy that despite the 
success rate of students who did attend the MLC more than once, Pell and Croft reported that roughly $10 \%$ of students visited the MLC less frequently (once or never) and failed and some of these students, i.e. those who had failed with an E grade, could have passed with more regular visits.

Mac An Bhaird et al. (2009) considered data for the MLC at Maynooth University and found significant differences in the pass rates between First Arts students taking mathematics who had attended the MLC more than once $(89 \%)$ and those who had attended once or never $(62 \%)$. To account for prior mathematical knowledge, the study grouped the students by the grade (A, B, C and D) achieved and level (Higher or Ordinary) undertaken for the Leaving Certificate (LC), the terminal examination taken at the end of secondary education in Ireland. When the authors considered the First Arts students, the mean mark in every subgroup was higher if they had attended the MLC more than once. For First Science students, the earlier results were not statistically significant but when diagnostic test scores were used to categorize at-risk students, there was a small but significant difference in pass rates as well as mean final mark between those who attended the MLC more than once and those who attended once or never.

A subsequent study for the same MLC by Berry et al. (2015) further validated the advantage to at-risk students who used the MLC in two academic years 2011/2012 and 2012/2013. This report used multiple regression analysis to identify the predictors of final mark among First Science students using their LC points (note: a certain number of 'points' are awarded by the Higher Education Institutes (HEIs) for the grade achieved in a student's best six subjects in the LC, with entry to higher education courses contingent on achieving a specified number of points), diagnostic test scores, number of visits to the MLC and the total duration spent in the MLC. The study found 
the LC points, diagnostic test scores and the total duration to be predictors for final mark in 2011/2012 and LC points, diagnostic test scores and total visits to be predictors in 2012/2013. To gain further insight, the paper also conducted a two-step cluster analysis from which it was found that the subgroup that performed least well in both years was the one which demonstrated low engagement with the MLC and had weak mathematical background.

Lee et al. (2008) also considered linear regression for predicting exam performance among engineering students. The significant predictors were found to be diagnostic test scores, whether a student had visited the MLC or not and how many statistics modules the students had taken at A-level. They did argue, however, that not all diagnostic test scores may be good predictors, as these vary widely in design, and that the model would not be directly transferrable to other diagnostic tests, but that, nonetheless, their diagnostic test scores were an efficient and reliable way of identifying at-risk students.

All of these papers recognize that accrediting student performance to engagement with mathematics support is non-trivial and other factors, which can be all but impossible to measure, are also predictors of student success. However, in a longitudinal study, with a large sample size, it is possible to investigate whether the contribution of mathematics support may be significant, as described in the next section.

\section{Methodology}

To add to and enhance the existing body of work, data for first year students who enrolled in first year Mathematics service modules from 2004/2005 to 2015/2016, inclusive, were collected. This data set included over 10,000 students who undertook one of the following modules, which will be identified by the labels Modules A-E in this paper: 
- IT Mathematics (Module A)

- Mathematics for Scientists (Module B)

- Calculus (Module C)

- Mathematics for Economics and Business (Module D)

- Accounting Mathematics 1 (Module E)

In addition, information on academic performance in these modules i.e. students' examination mark, final mark and examination result (pass, fail, etc.) and their prior mathematics background i.e. LC grade in Mathematics, the level at which they studied Mathematics (Higher or Ordinary) and their overall LC points were collected. The data also provided information on their course choice, department and faculty.

From the MLC, data were collected on student attendance, the module for which they sought assistance, how many refresher and revision classes students attended (where relevant) and their diagnostic test scores (where available). These sources of information were collated to create the data set that has been used in this report. The data was inputted into Statistical Package for the Social Sciences (SPSS) and this was used for all the statistical tests undertaken.

Initially, some generic analysis of how the student profile has changed was conducted. This included comparing the number of Higher Level (HL) with Ordinary Level (OL) students, mean final mark, attendance at the MLC, etc. Subsequently some initial correlation tests were conducted to assess if there was any relationship between attendance at the MLC and the final mark as a way to validate applying regression analysis, which was the last step. Binary logistic regression was applied to the data set to investigate if and how much the MLC has an impact on pass rates.

The independent variables that were included in the regression were diagnostic score, LC points, total visits at the MLC, LC grade and final examination outcome. 
Diagnostic test scores were categorized in bands of 10 percentage points, but the first four categories were merged into two due to the low number of students in these bands, meaning that they were grouped $0-19,20-39,40-49,50-59$ and so on. The total number of visits to the MLC were grouped as $0,1,2-5,6-10,11-14$ and 15 or greater, for reasons that will be explored in the next section. For the LC grade, students were categorized into Higher $(\mathrm{H})$ or Ordinary $(\mathrm{O})$ level, and then each level was split into A, $\mathrm{B}, \mathrm{C}$ or $\mathrm{D}$, representing the grade category achieved by the student. (In fact, each grade is subdivided, with A split into A1 and A2 and B, C and D split in three e.g. B1, B2, B3 and so on, but these grades were merged to avoid an excessive number of categories with a low number of entries.) In addition, $\mathrm{HA}$ and $\mathrm{HB}$ were combined, as were OC and $\mathrm{OD}$, as the numbers in the extreme categories were too low for accurate analysis otherwise. Those not fitting into these categories were deemed to be missing data points.

While over 10,000 students enrolled in at least one of the above modules, there are data missing for various reasons. For example, every year a certain portion of students do not take the diagnostic test as it is not mandatory. From the central data set, there were some students who did not have a LC grade, LC points or level at which Mathematics was taken at LC (e.g. international students or mature students) and these will be treated as missing data and left out of the analysis where necessary. Every effort has been made to ensure that the maximum number of data points is used in the following analysis.

\section{Results and Discussion}

\subsection{Student background 2004/2005 - 2015/2016}

The data included information on 10,504 students over 12 years, from the academic 
years 2004/05 to 2015/2016. Descriptive statistics on student intake is presented below. Figure 1 shows the total number of students enrolled in a first-year service mathematics module in DCU each year.

This was further categorised into the individual modules involved, which is presented in Figure 2. The fluctuation in module numbers from year to year is dependent upon the intake into certain programmes as well as the movement of programmes from one module to another if some redesign of the programme had taken place.

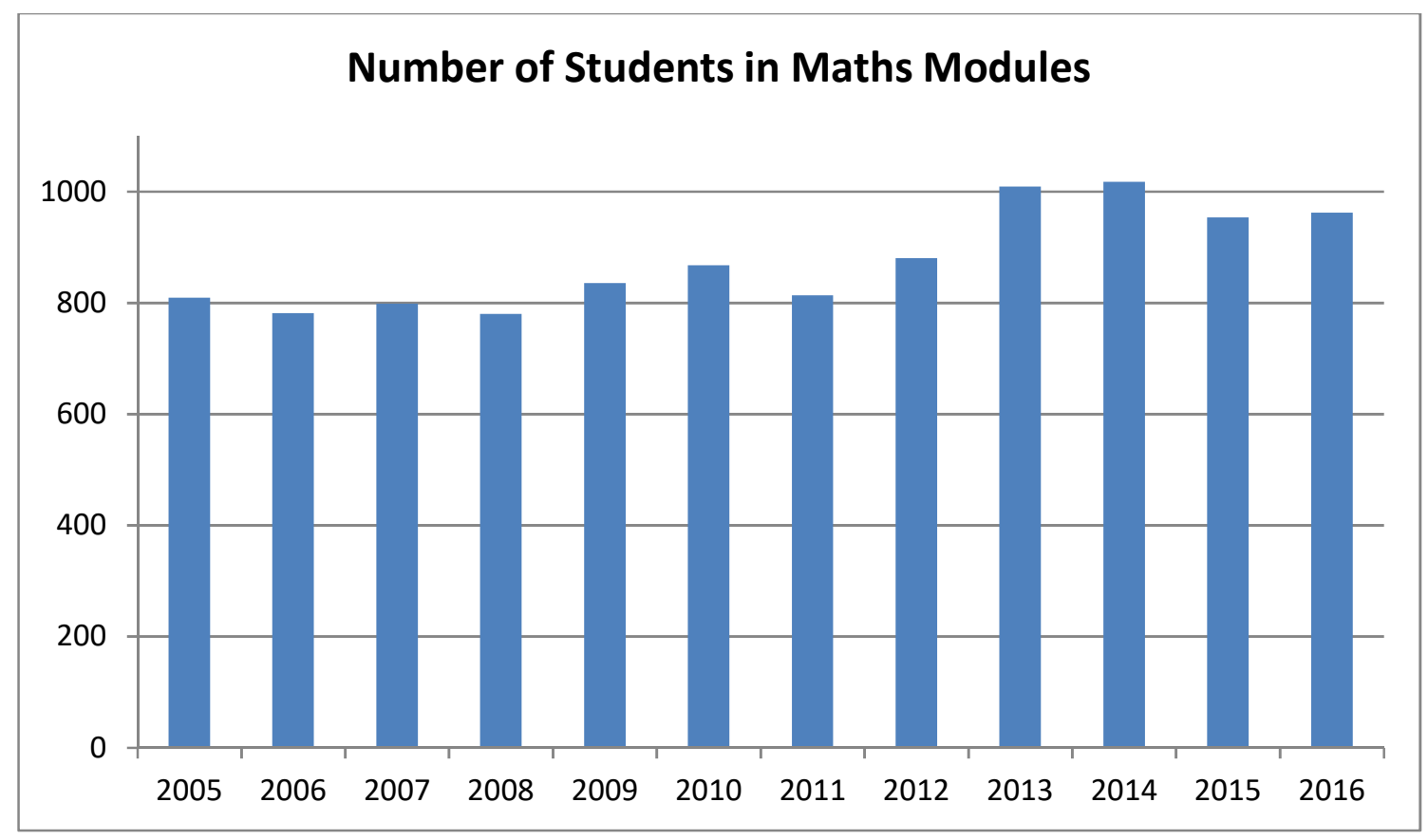

Figure 1: Number of students registered in first-year service mathematics modules from the academic years 2004/2005 (shown as 2005) to 2015/2016 (shown as 2016). 


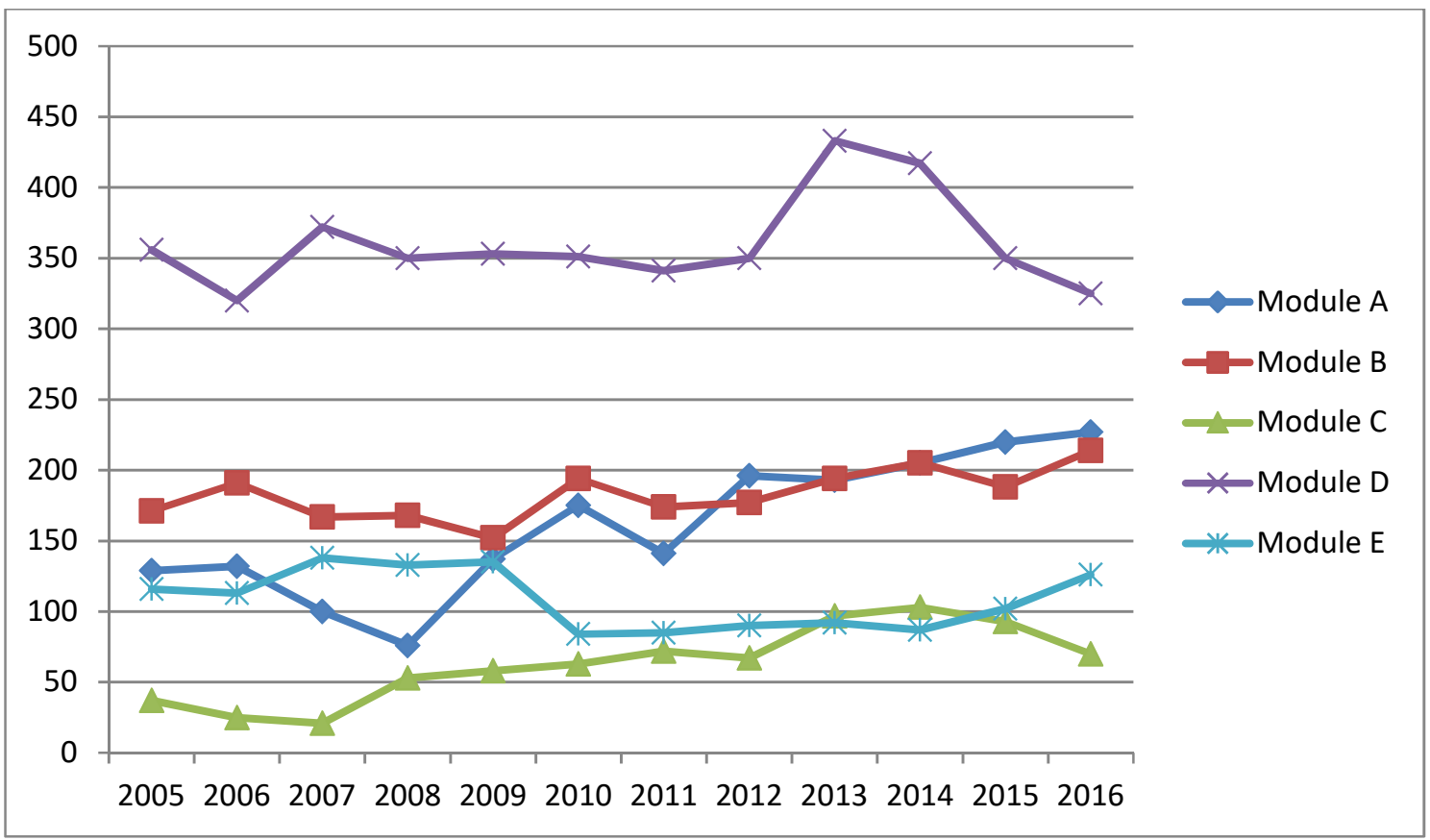

Figure 2: Breakdown of student numbers by module for each of the first-year service mathematics modules from 2004/2005 - 2015/2016.

Categorising students by the grade they achieved in LC Mathematics and the level at which they took the examination, there are clear changes in profile. For example, the number of students achieving a HD and $\mathrm{HC}$ has increased since the academic year 2012/2013 (labelled 2013 in Figure 3). 


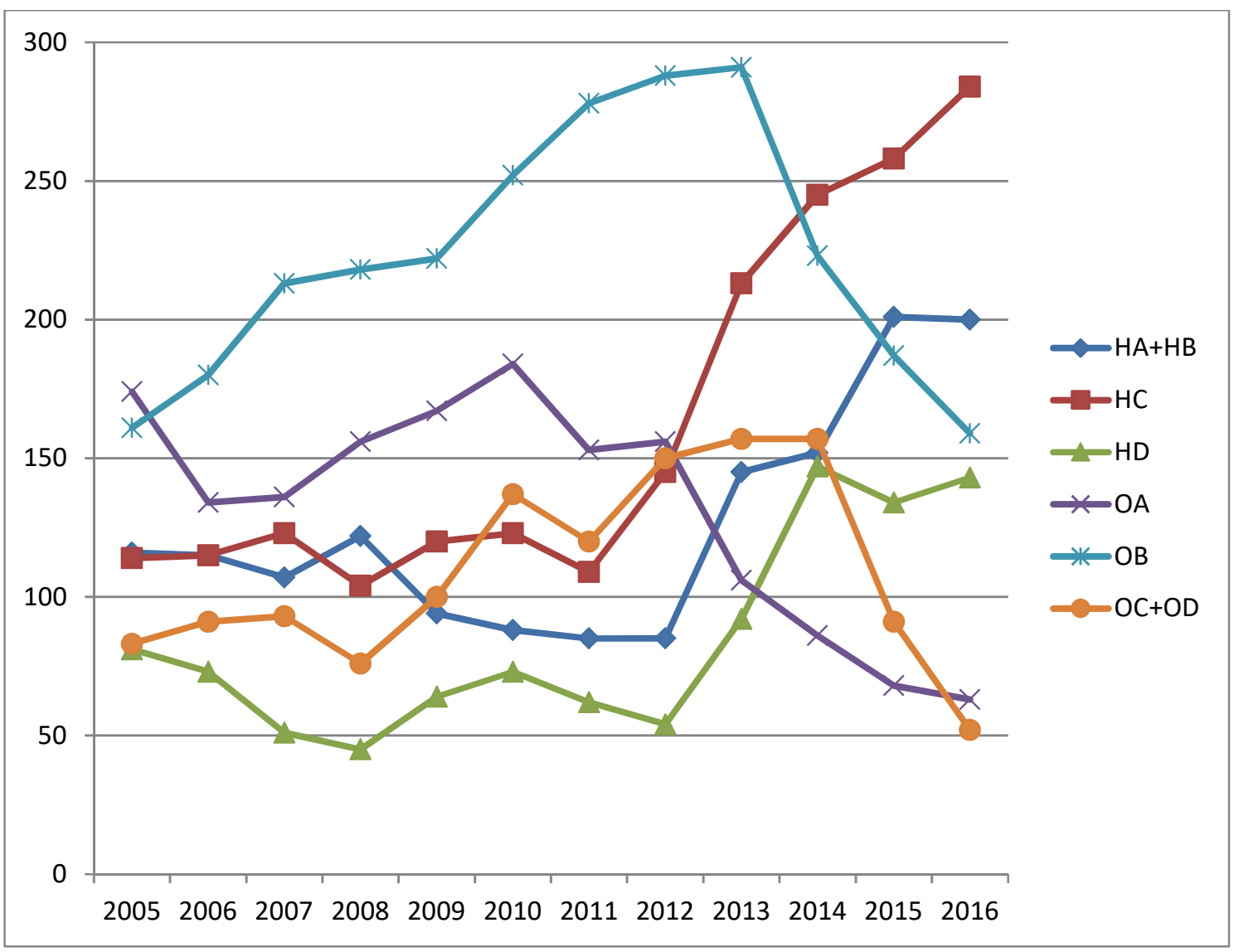

Figure 3: Profile of LC Mathematics levels and grades for incoming service mathematics students from 2004/2005 - 2015/2016, where H represents Higher Level and $\mathrm{O}$ represents Ordinary Level.

This is a response that has been observed nationwide and is likely to be due to an initiative whereby 25 bonus points have been awarded by all HEIs to all students who pass HL Mathematics since 2013. Equivalently, the number of OA and OB students dropped as these are the cohort of students most likely to be incentivised by the bonus points to take the Higher Level paper instead of Ordinary Level.

In line with this, there have been changes in the absolute number of students enrolling in DCU first-year service mathematics modules who have studied mathematics at Higher or Ordinary levels, as shown in Figure 4. 


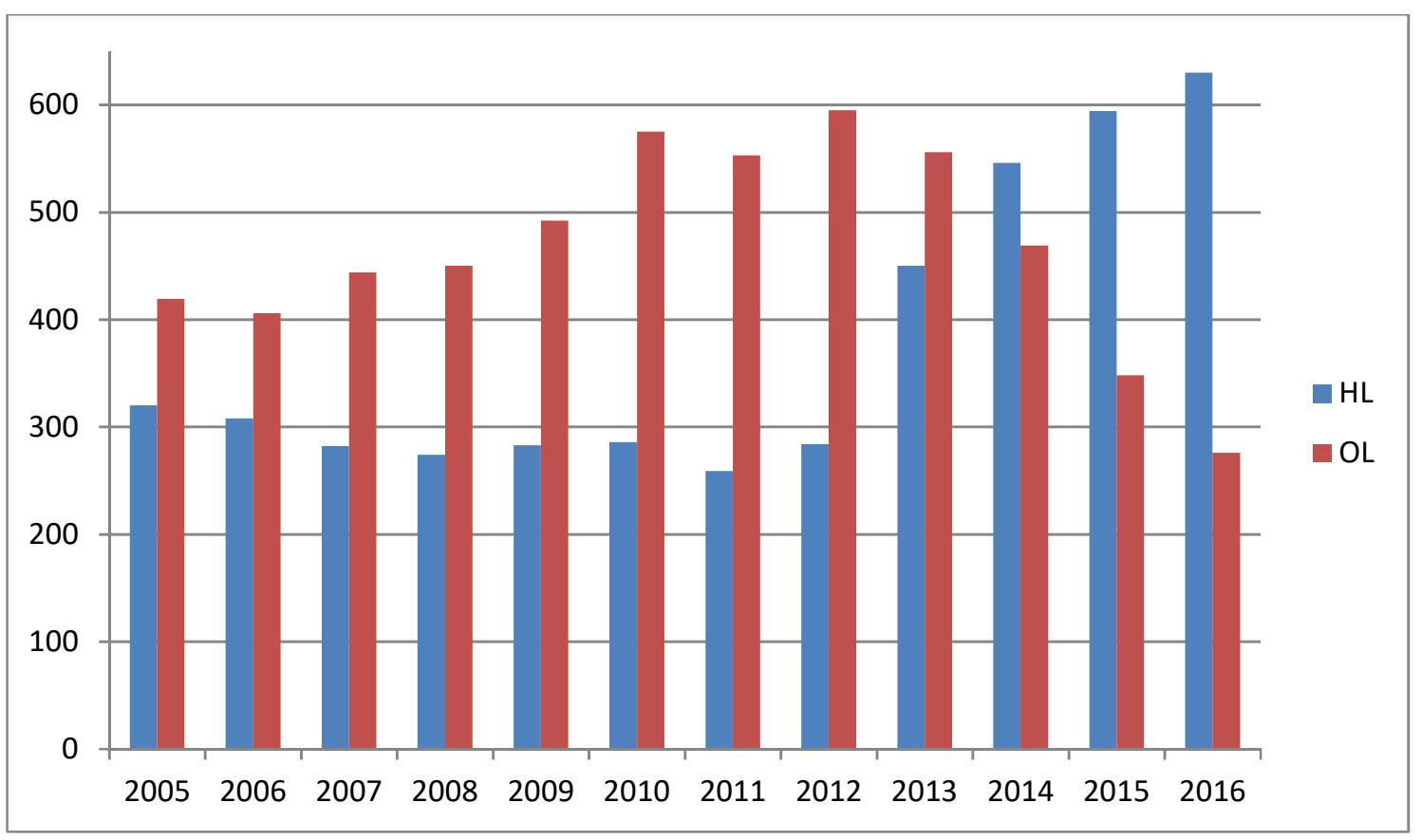

Figure 4: Number of incoming service mathematics students who took Higher Level (HL) or Ordinary Level (OL) mathematics for Leaving Certificate from 2004/2005 $2015 / 2016$.

Before 2013/14, OL students outnumbered HL students but in the last three years, this trend has reversed. There was also a large increase in the HL students in 2012/2013 which marked the first year of the bonus points. This profile is again representative of the national picture.

All first-year service-mathematics students in DCU are also given a mathematics diagnostic test during Orientation Week, the results of which are shown in Figure 5. It should be noted that there was no diagnostic test in 2013/2014, and also that the marking scheme changed in 2007/2008 to introduce negative marking. This was done with the aim of providing a more accurate assessment of student knowledge, as the multiple-choice test without penalties which was used prior to $2007 / 2008$ may have artificially inflated some students' marks, which is backed up by the fact that the number of students in the $80-100 \%$ band dramatically reduced once negative marking was brought in. 


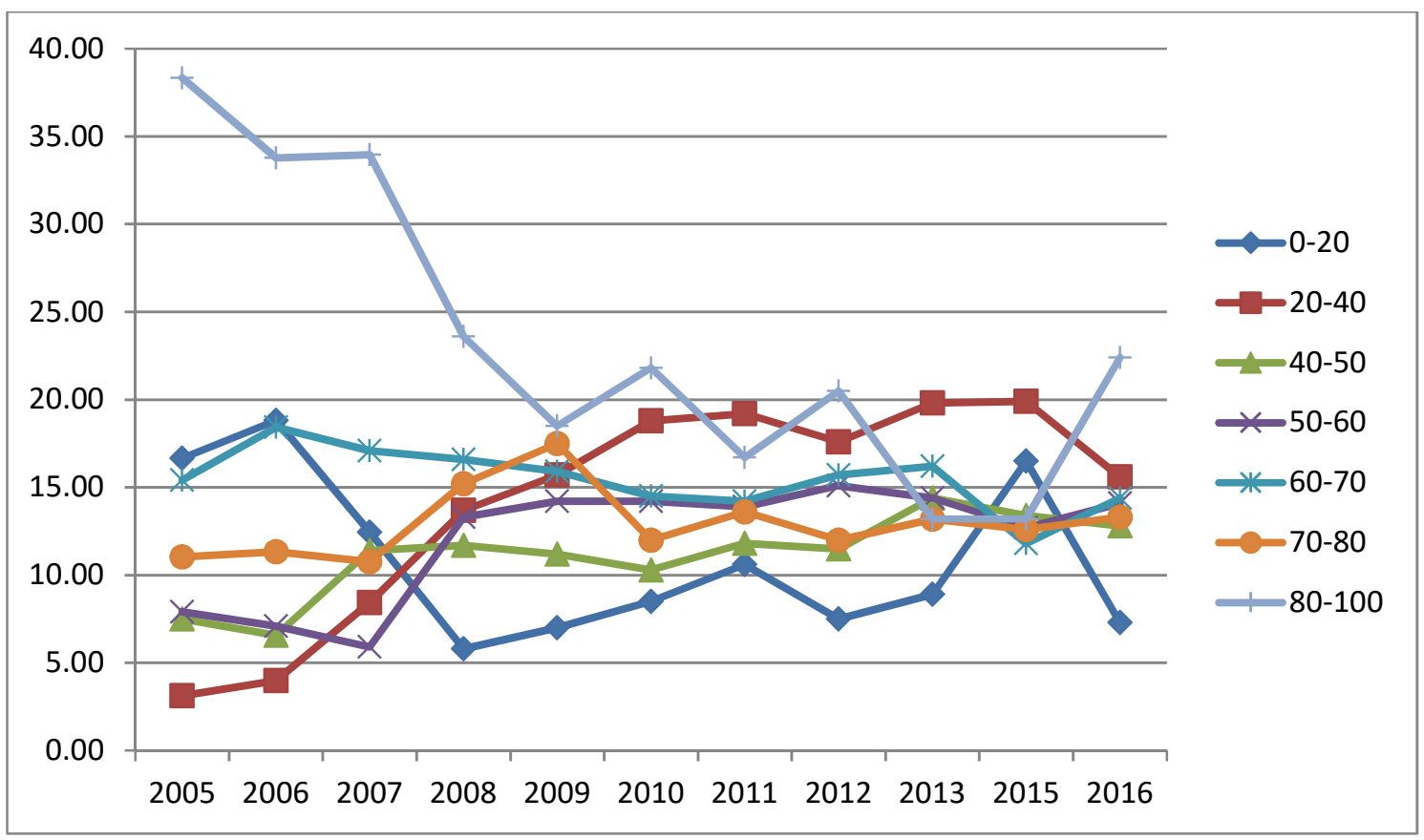

Figure 5: Percentage of incoming service mathematics students who achieved certain diagnostic test marks during Orientation Week from 2004/2005 - 2015/2016. Note that no data is available for $2013 / 2014$, as the diagnostic test did not take place that year.

\subsection{Student examination performance 2004/2005 - 2015/2016}

To also gain a better understanding of student examination performance, the changes in pass rates over this period were also considered, as shown in Figure 6. This figure excludes students who did not sit their final examinations due to illness or other reasons, roughly $2 \%$ on average. 


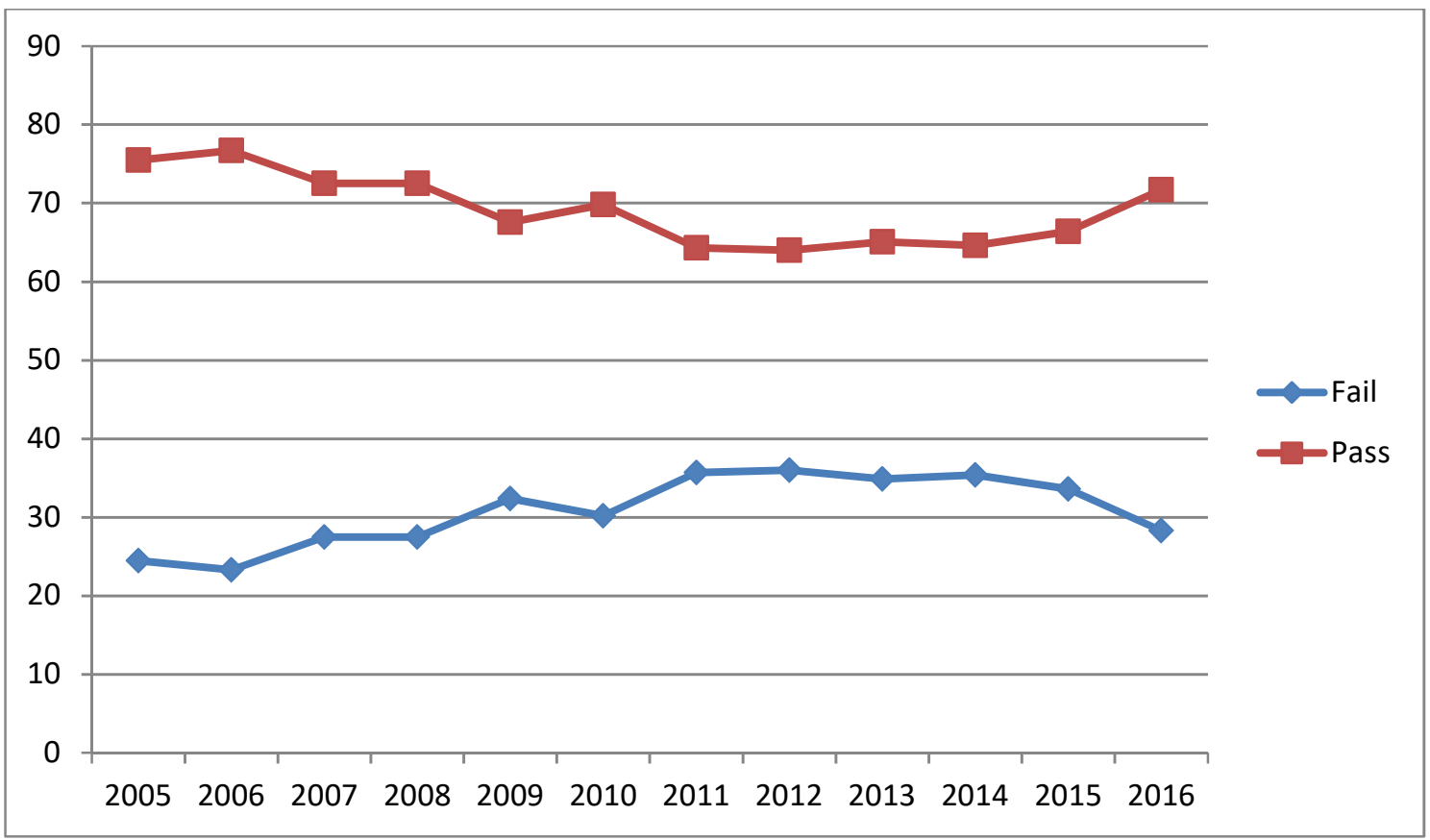

Figure 6: Pass and fail rates for first-year service mathematics modules from 2004/2005 $-2015-2016$.

If these pass rates are broken down by module, as shown in Figure 7, a variable picture emerges, depending on the module in question, with Module $\mathrm{C}$ appearing particularly changeable, but it must be borne in mind that in many years, the student numbers in this module were almost ten times smaller than in the largest module considered (Module D), so such fluctuations are to be expected with smaller sample sizes. 


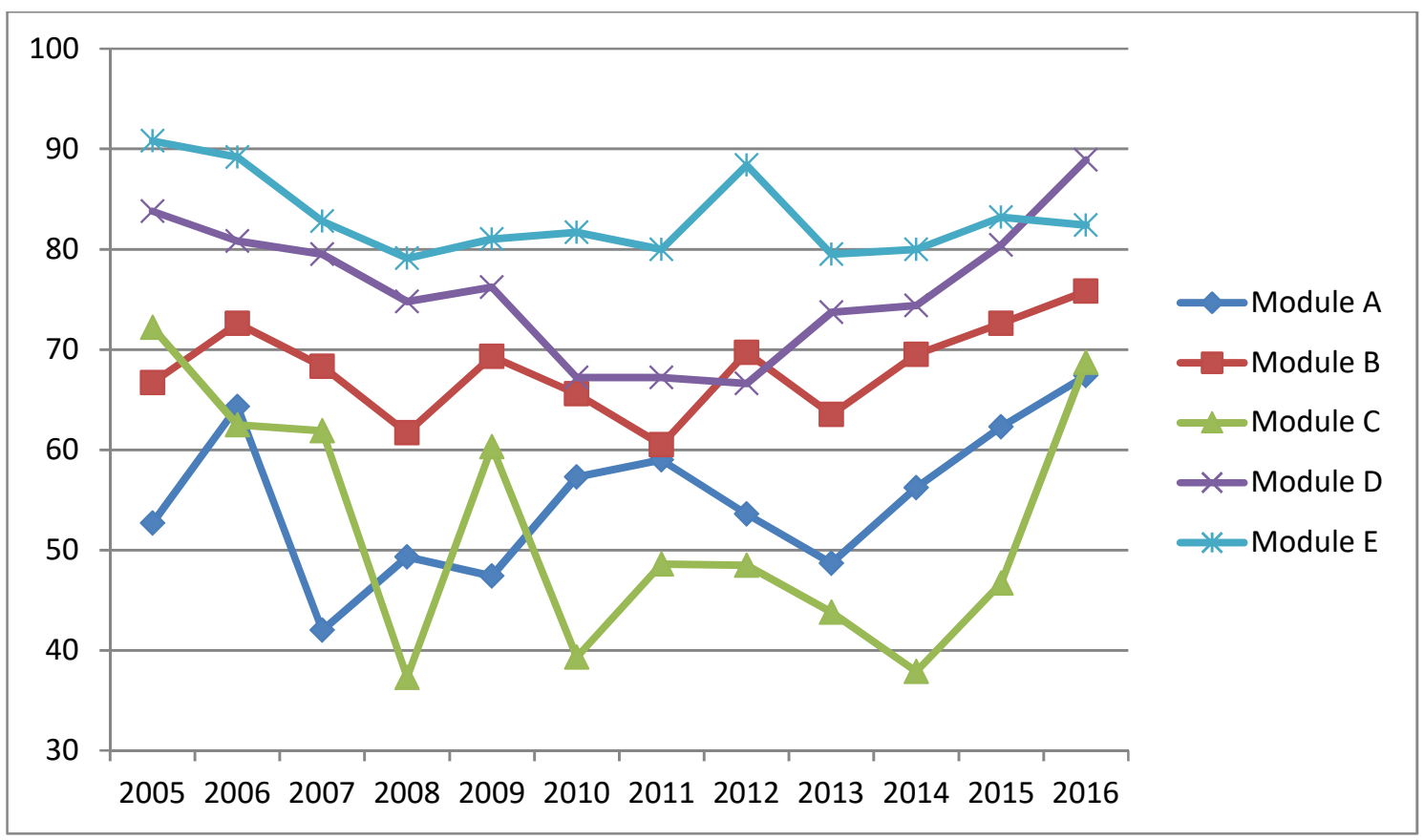

Figure 7: Pass rates by first-year service mathematics module from 2004/2005 - 20152016.

\subsection{Student engagement with mathematics support 2004/2005 - 2015/2016}

Attendance at the MLC from first-year service-mathematics students fluctuated vastly during the 12 years analysed here, with numbers ranging from around 500 to 1,500 visits in a year. These variations were due to a number of factors, ranging from raw student numbers in any given year to the types and number of assessments in certain modules (as peaks in attendance are usually noticed immediately prior to in-class tests, particularly in larger modules).

In order to analyse the potential impact of mathematics support upon individual students, it was necessary to categorise students by the number of visits they made to mathematics support. As mentioned in the previous section, in order to address our second research question as to whether or not a single visit to mathematics support can have an impact upon student examination performance, students who did not engage 
with mathematics support in any way were categorised differently from those who visited once, as shown in Figure 8.

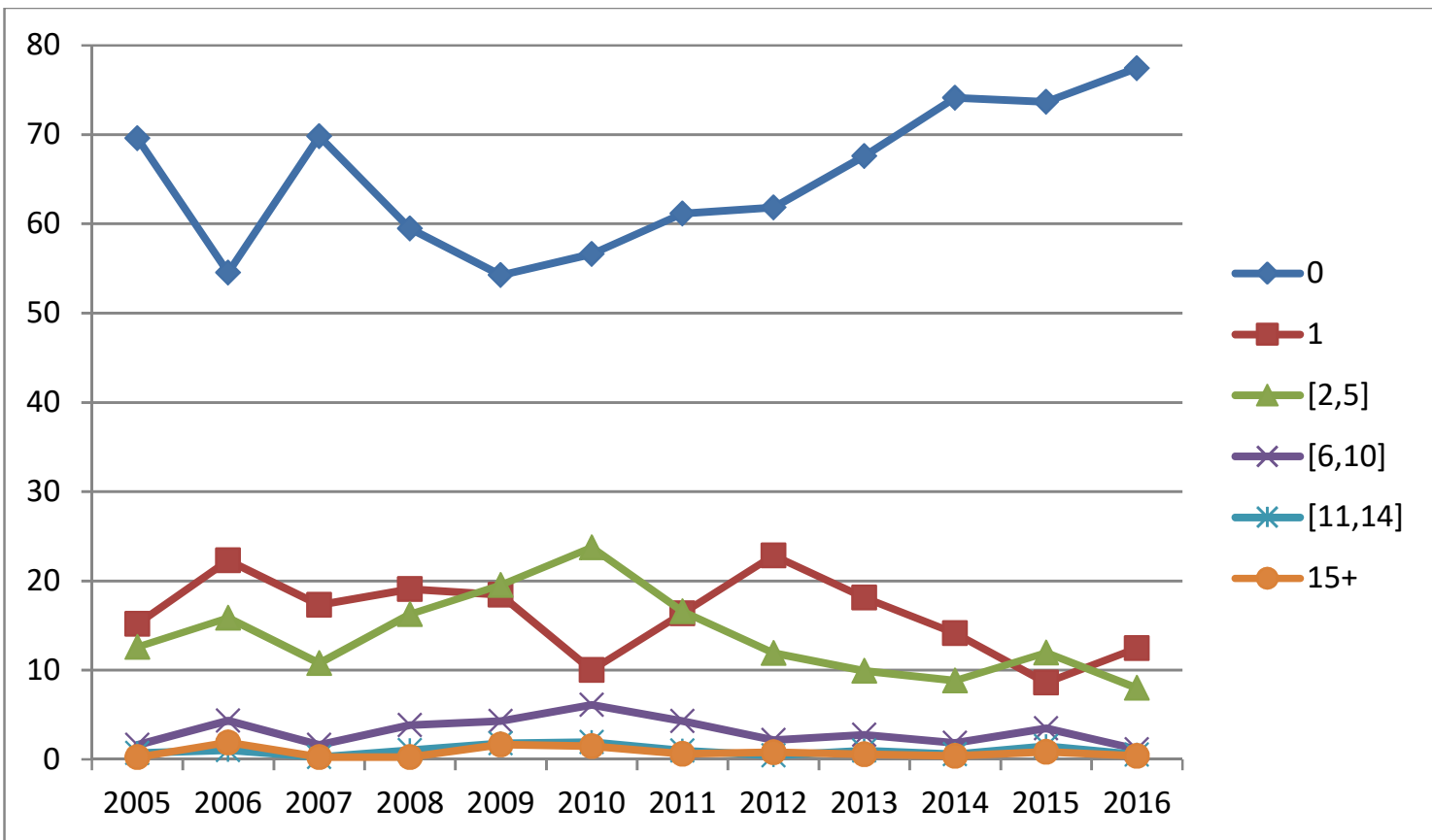

Figure 8: Percentage of first-year service mathematics students who made 0, 1, 2-5, 6$10,11-14$ and $15+$ visits to the MLC in any given year.

This does not, however, convey much about the prior mathematical attainment of students who made these visits, and so the numbers at each LC grade level who attended the MLC were calculated and are shown in Figure 9. This is a valuable way of looking at this data as it allows us to account for both engagement with the MLC and prior knowledge, as well as quantifying the number of students of each background that are regularly attending for additional support. From this, it can be seen that the largest numbers of students attending are all from Ordinary Level cohorts, with OB by far the highest across the visit categories. 


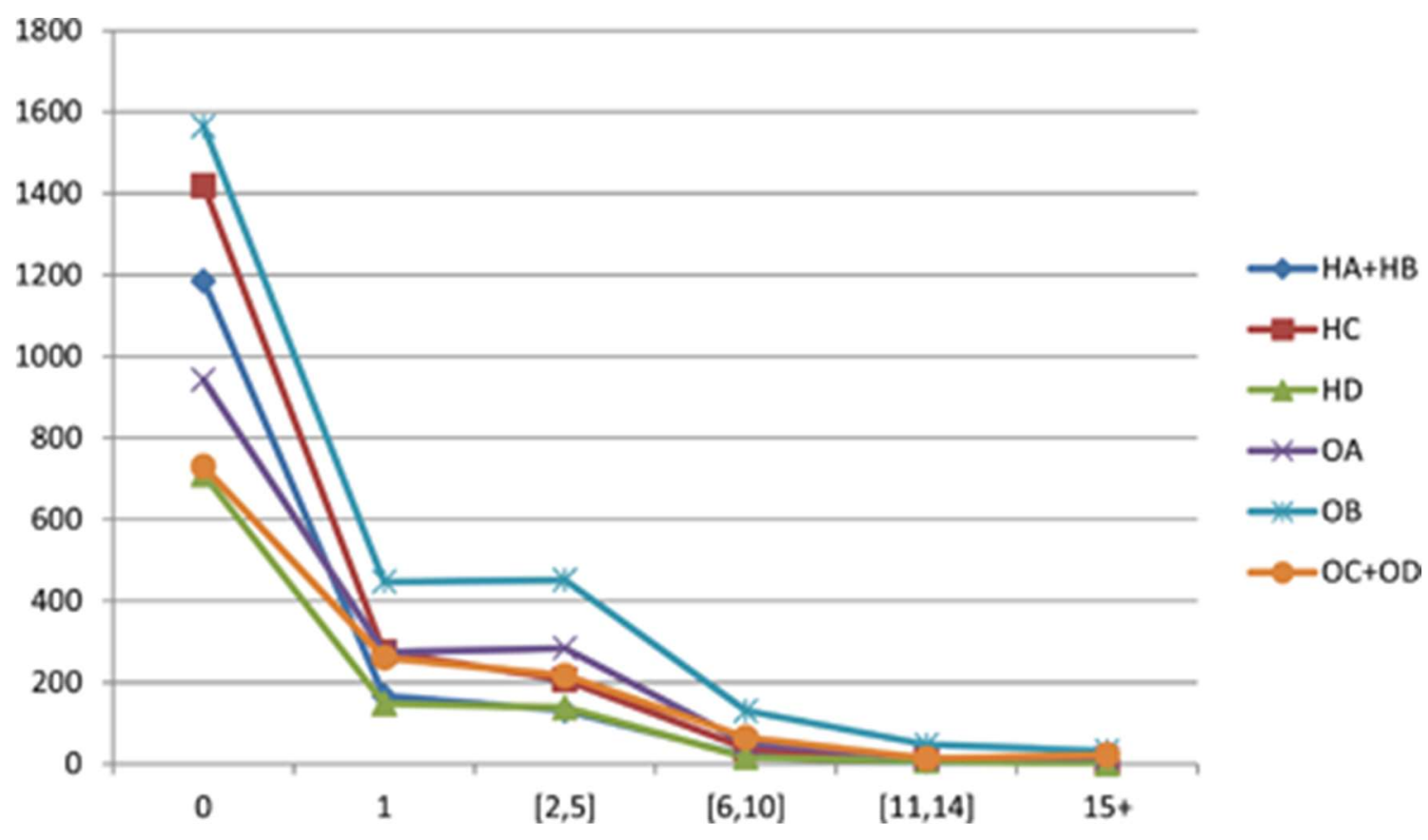

Figure 9: Number of students in each LC grade category who made a set number of visits to mathematics support.

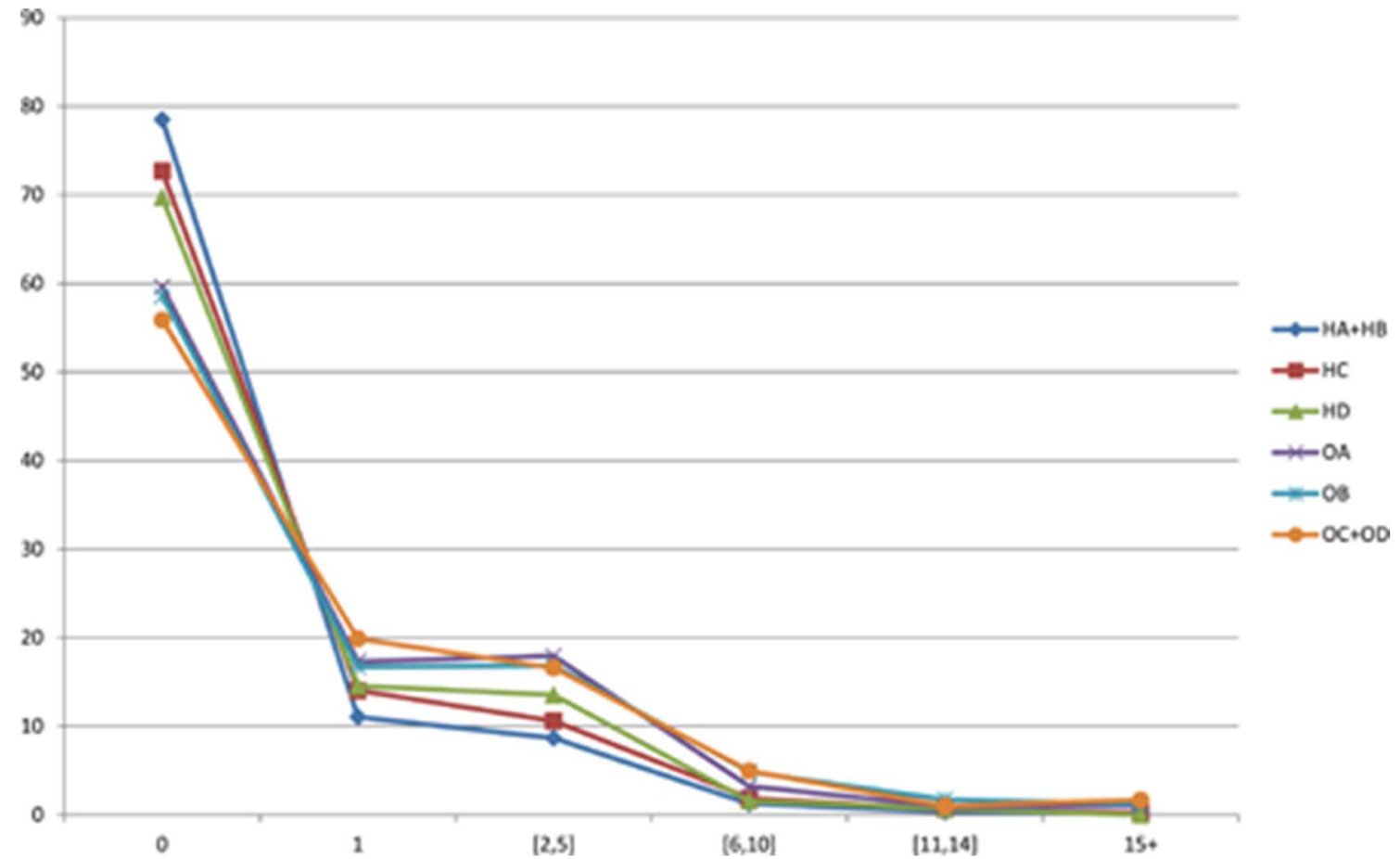

Figure 10: Percentage of students in each LC grade category (expressed as a percentage of all students in that LC grade category) who made a set number of visits to mathematics support. 
Another important angle is to look at attendees as a percentage of the total number of students at each LC grade level, as shown in Figure 10. From this, it can be seen that the spread of attendance by LC grade is as might be expected, with the highest percentage of non-attendees from the HA+HB bracket, and the lowest percentage from the $\mathrm{OC}+\mathrm{OD}$ bracket. This trend then reverses for a single visit to the MLC and continues almost uniformly through the greater number of visits, with the highest percentage of attendees from the $\mathrm{OC}+\mathrm{OD}$ cohort (as a percentage of all students in the $\mathrm{OC}+\mathrm{OD}$ grouping) and the lowest from $\mathrm{HA}+\mathrm{HB}$.

\subsection{Statistical analysis of results}

To establish if there was a relationship between final mark and attendance at the Maths Learning Centre, the sample Pearson correlation coefficients between the two for each LC grade was done. Significant correlation coefficients were found for grades between $\mathrm{HD}$ and $\mathrm{OC}+\mathrm{OD}$, and these are presented in Table 1.

\begin{tabular}{|l|c|c|c|c|}
\hline LC Grade & HD & OA & OB & OC+OD \\
\hline Pearson Correlation & $0.069^{*}$ & 0.078 & 0.169 & 0.244 \\
\hline
\end{tabular}

Table 1: Significant correlation coefficients when final mark was correlated with attendance at the Maths Learning Centre. * Significant at the 0.05 level. Others are significant at the 0.01 level.

In the former analyses, prior mathematical attainment was taken into account by grouping the students into their LC grades and levels. However, this type of model would consider a student who achieved a final mark of $87 \%$ to be fundamentally different in their attainment level from a student who received $88 \%$ or $86 \%$ which is, for the purpose of this study, an excessively fine-grained distinction. Thus in the logistic regression described below, final marks will simply be categorised into a pass or a fail, 
allowing the use of binomial logistic regression, while also providing us with valuable information about the potential of mathematics support to impact upon student success in passing mathematical modules.

The binomial logistic regression model used is defined as follows: Let $y_{i}$ be an indicator of whether student $i$ passed their module such that $y_{i}=1$ if passed and $y_{i}=0$ if not passed. The model is then defined in terms of the log-odds of student $i$ passing the model as follows:

$$
\ln \left(\frac{P\left(Y_{i}=1\right)}{P\left(Y_{i}=0\right)}\right)=X_{i} \beta
$$

where $\boldsymbol{X}_{i}$ denotes row $i$ of a matrix whose columns contain the responses for the dependent variables which are multiplied by a vector of model parameters denoted by $\beta$. The dependent variable in the model was whether or not student $i$ passed their module. Since this model presents the log-odds as a linear model as show in the equation, as is usual practice we report, in Table $2, \exp (\beta)$, the exponential of the estimated parameters (odds ratios) which represent the multiplicative effect of the independent variables on the odds of passing a module. 
Table 2: Table showing how the odds of a student passing their module are higher if they have (a) attended the MLC [total visits] times than a student who has never attended the MLC, assuming LC grade and module studied are kept constant and (b) if they have received [LC Grade] in their terminal mathematics examination in school than a student who received an OC or OD, assuming number of visits to the MLC and module studied are kept constant

\begin{tabular}{lll} 
& $\begin{array}{l}95 \% \text { confidence interval for } \\
\operatorname{Exp}(B)\end{array}$ & $\begin{array}{l}\text { p- } \\
\text { value }\end{array}$ \\
& Lower & Upper \\
\hline
\end{tabular}

\section{Total visits}

\begin{tabular}{lllll}
\hline 1 & 1.632 & 1.348 & 1.976 & $<0.001$ \\
\hline $2-5$ & 2.410 & 1.977 & 2.938 & $<0.001$ \\
\hline $6-10$ & 4.301 & 2.962 & 6.246 & $<0.001$ \\
\hline $11-14$ & 6.350 & 3.178 & 12.690 & $<0.001$ \\
\hline $15+$ & 13.778 & 6.279 & 30.231 & $<0.001$ \\
\hline LC Grade & & & & $<0.001$ \\
\hline HA+HB & 164.012 & 96.798 & 277.896 & $<0.001$ \\
\hline HC & 34.250 & 24.629 & 47.630 & $<0.001$ \\
\hline HD & 12.265 & 8.984 & 16.744 & $<0.001$ \\
\hline OA & 3.501 & 2.788 & 14.592 & $<001$ \\
\hline OB & 11.077 & 8.408 & & \\
\hline
\end{tabular}

CAO Points

$\begin{array}{llll}1.001 & 1.001 & 1.002 & <0.001\end{array}$




\section{Diagnostic}

Scores

\begin{tabular}{|c|c|c|c|c|}
\hline$[20,30)$ & 1.636 & 1.174 & 2.279 & 0.004 \\
\hline$[30,40)$ & 1.317 & 0.961 & 1.805 & 0.086 \\
\hline$[40,50)$ & 1.149 & 0.847 & 1.558 & 0.371 \\
\hline$[50,60)$ & 1.604 & 1.179 & 2.182 & 0.003 \\
\hline$[60,70)$ & 1.912 & 1.399 & 2.614 & $<0.001$ \\
\hline$[70,80)$ & 1.777 & 1.261 & 2.504 & 0.001 \\
\hline$[80,90)$ & 3.359 & 2.281 & 4.947 & $<0.001$ \\
\hline$[90,100]$ & 3.212 & 2.000 & 5.159 & $<0.001$ \\
\hline \multicolumn{5}{|l|}{ Module } \\
\hline Module A & 0.710 & 0.525 & 0.960 & 0.026 \\
\hline Module B & 0.732 & 0.548 & 0.978 & 0.035 \\
\hline Module C & 0.096 & 0.068 & 0.136 & $<0.001$ \\
\hline Module D & 2.128 & 1.589 & 2.849 & $<0.001$ \\
\hline
\end{tabular}

Several possible regression models were considered with the following independent variables: diagnostic test scores, total visits to the MLC (in categories), LC mathematics grade, LC points and mathematics module taken. It had been feared that there would be a high correlation between LC grade and the diagnostic test scores but when Variance 
Inflation Factors were calculated for all independent variables, these were all lower than two, where only scores above 10 would be a general cause for concern in terms of multicollinearity. As a result, the full model was used for this analysis, and it was found that every category in each independent variable was significant. This model is a significantly better fit to the data than the null model (chi-square $=1975.335, \mathrm{df}=$ 23, $p<0.001$ ), with $79.9 \%$ correct classification (compared with $70 \%$ for the null model). The Hosmer and Lemeshow test for the goodness of fit also suggests that the model is a good fit to the data as $p=0.535(>0.05)($ chi-square $=7.014, \mathrm{df}=8)$. Nagelkerke's R22 suggests that the model explains roughly $42.4 \%$ of the variation in the outcome, which is acceptably good. Table 2 shows how much higher the odds of passing a module are for a student who engages a set number of times with mathematics support than for one who does not, as well as how much higher these odds are for students with a certain prior mathematical background over those with an OC or OD. Without a doubt, prior mathematical knowledge showed the largest impact by far in terms of the likelihood of a student not failing their module, as might have been expected. The odds of passing a module were roughly 3.5 times higher for a student with an OB compared with somebody with an OC or less with all else being equal. However, engagement with mathematics support also emerged as a significant influence upon examination outcomes. The odds of a student who only attended the MLC once passing their module were 1.63 times higher than for one who had never attended the MLC, assuming all other factors kept constant. The odds for a student who engaged frequently (15+ visits) passing were almost 14 times higher. The impact of the LC points was minimal overall, while the module in question and the diagnostic test scores did have a significant impact, as may have been expected. The fact that LC points did not emerge as a major predictor is not a surprise in that these are based on a student's 
best six subjects at LC, which may not include mathematics at all. The diagnostic test is nowhere near as clear a predictor as the LC grade, but previous research (Cleary, 2007) has shown that a second, later test tends to be more closely correlated with eventual performance, as those who engage with study and support after an initial low score often end up doing well overall.

\section{Conclusions}

We set out in this research paper to consider two main research questions. The first of these involved controlling for factors such as prior mathematical background and module studied and investigating the potential impact of usage of the mathematics support service upon student success in final examinations. The results of the binomial logistic regression on 12 years of data from the MLC in DCU shows that even a single visit to the MLC can have a positive impact upon a student's chance of passing their first-year service mathematics module, with all other factors being equal. Over the course of those 12 years, we have seen fluctuations in attendance patterns from various modules, largely due to factors outside the control of mathematics support such as student numbers, prior mathematical background, changes in assessment patterns and so on. As a result, taking a longitudinal approach to such data provides us with more robust findings to reinforce the value of mathematics support in higher education.

Our second research question considered whether it may be appropriate to consider even a single visit as engaging with mathematics support, given the varying levels of support that may be optimal for different students. As a result, for our data set, unlike previous research in this area, we considered students who had made a single visit to mathematics support as being different from those who had not attended at all, and our statistical analysis backed up this decision. We originally explored this as a result of anecdotal evidence of students attending with one particular area of difficulty 
and devoting a couple of hours in the centre to overcoming this but not subsequently returning. It would appear that for some such students, this one visit was still of significant help to them, an important point to note for those involved in quantitative analysis of mathematics support data. It would be interesting to see if this result can be replicated in other centres around Ireland and UK as it is likely to have an effect upon the future reporting of the impact of mathematics support upon student retention and pass rates in mathematics modules.

BERRY, E., MAC AN BHAIRD, C. and O'SHEA, A., 2015. Investigating relationships between the usage of Mathematics Learning Support and performance of at-risk students. Teaching mathematics and its applications, 34(4), pp. 194-204.

CLEARY, J. (2007) Diagnostic testing - an evaluation 1998-2007. Proceedings of Second National Conference on Research in Mathematics Education (MEI2), 14th-15th September 2007. Drumcondra, Dublin: St. Patrick's College, pp. 216 228.

DOWLING, D., and NOLAN, B. (2006) Measuring the effectiveness of a maths learning support centre - the DCU experience. Proceedings of CETL-MSOR Conference 2006, 11 th - 12th September 2006. The Maths, Stats and OR Network: Birmingham, pp. 51-54. http://www.mathcentre.ac.uk/resources/uploaded/cetl-msor2006conferenceproceedingsweb.pdf.

LEE, S., HARRISON, M.C., PELL, G. and ROBINSON, C.L., 2008. Predicting performance of first year engineering students and the importance of assessment tools therein. Engineering Education: A Journal of the Higher Education Academy, 3(1), pp. 44-51.

MAC AN BHAIRD, C., MORGAN, T. and O'SHEA, A., 2009. The impact of the mathematics support centre on the grades of first year students at the National University of Ireland Maynooth. Teaching Mathematics and Its Applications, 28(3), pp. 117-122.

MATTHEWS, J., CROFT, T., LAWSON, D. and WALLER, D., 2013. Evaluation of mathematics support centres: a review of the literature. Teaching Mathematics and its Applications, 32(4), pp. 173-190. 
PATEL, C. and LITTLE, J., 2006. Measuring maths study support. Teaching Mathematics and its applications, 25(3), pp. 131-138.

PELL, G. and CROFT, T., 2008. Mathematics support—support for all? Teaching mathematics and its applications, 27(4), pp. 167-173. 